\title{
Hookworm-like eggs in children's faecal samples from a rural area of Rwanda.
}

\author{
María José Irisarri-Gutiérrez ${ }^{1}$, Carla Muñoz-Antolí1 ${ }^{1}$ Lucrecia Acosta ${ }^{2-3}$, Lucy Anne Parker ${ }^{4}$, \\ Rafael Toledo ${ }^{1}$, Fernando Jorge Bornay-Llinares², José Guillermo Esteban ${ }^{1}$
}

1. Área de Parasitología del Departament de Biología Cel.lular i Parasitología, Facultat de Farmàcia, Universitat de València, Valencia, Spain.

2. Área de Parasitología del Departamento de Agroquímica y Medioambiente, Facultad de Farmacia, Universidad Miguel Hernández de Elche, Alicante,Spain.

3. Laboratorio de Análisis Clínicos, Sanatorio "Fontilles", Vall de Laguar, Alicante, Spain.

4. Departamento de Salud Pública Historia de la Ciencia y Ginecología, Universidad Miguel Hernández de Elche, Alicante, Spain.

\begin{abstract}
Background: Hookworm eggs identification and quantification is usually carried out by Kato-Katz method. However various structures present in the smear may be confused with eggs of such parasites.

Objective: To document the presence of structures in Kato-Katz slides that could initially be misinterpreted as hookworm eggs.

Method: 497 faecal samples were analysed by Kato-Katz technique, diphasic concentration technique, agar-plate coprocultive and larvae obtained were analysed by PCR and characterized by sequencing.

Result: Hookworm-like eggs were found in $159(32 \%)$ of the samples by Kato-Katz, finally identified as Caenorhabditis elegans by PCR technique.

Conclusion: The diagnosis of human hookworm eggs, only by the use of Kato-Katz technique can lead to false positives because of similarities with eggs of other free-living worms, from wet soils like those of Rwanda that could contaminate stool samples.

Keywords: Hookworm eggs, Kato-Katz method, misclassification, Rwanda.

DOI: http://dx.doi.org/10.4314/ahs.v16i1.11

Cite as: Irisarri-Gutiérrez MJ, Muñoz-Antoli C, Acosta L, Parker LA, Toledo R, Bornay-Llinares FJ, Esteban JG. Hookworm-like eggs in children's faecal samples from a rural area of Rwanda. Afri Health Sci. 2016;16(1): 83-88. http:/ / dx.doi.org/10.4314/abs.v16i1.11
\end{abstract}

\section{Introduction}

Soil Transmitted Helminths (STH) infect more than 1 billion people worldwide and is an enormous problem of public health in tropical countries ${ }^{1}$. A national survey in Rwanda gave data of $66 \%$ prevalence of STH, being hookworm infection of about $31 \%{ }^{2}$.

One of the most commonly stool examination techniques applied for detection of STH ova in stools is
Corresponding author:
Maria José Irisarri Gutiérrez, Departament de Biología Cel.lular i Parasitología, Facultat de Farmàcia, Av. Vicent Andrés Estellés, s/n 46100, Burjassot, Valencia, Spain
Tel +34963543149
Fax +3496354476
Email: maria.jose.irisarri@uv.es

the Kato-Katz technique ${ }^{3-5}$ because of its easy use in the field, its low cost and its non-invasive nature. For those reasons, the Kato-Katz technique is the method of choice in parasitological surveys ${ }^{6,7}$. However, for the detection of hookworms this technique has a relatively poor sensitivity ${ }^{5}$, this may occur, because of the rapid clearing of hookworm eggs with time ${ }^{5,8}$. A long period from ejection of faeces until the preparation of slides, together with the time from Kato-Katz preparation until the beginning of the microscope examination, makes this technique less sensitive ${ }^{4}$. In addition, another problem is the similar shape of hookworm eggs with other structures, diverse in nature, which may lead to erroneous diagnoses.

The purpose of this report was to document the presence of eggs in Kato-Katz slides that could initially be misinterpreted as hookworm eggs.

\section{Methods}

Samples: In this cross-sectional study a total of 497 faecal samples from a population of children (aged be- 
tween 6 and 18 years old; mean: 11.0; SD: 2.32) from the rural area of Nemba Sector, that it is placed in the $\mathrm{Ga}-$ kenke District (located at an altitude between 1.700 and 2.700 meters), on the Northern Province of Rwanda, were collected during the dry season between August and September of 2011. For the samples collection, children were instructed to place the sample directly on the plastic collection tube. Samples were analysed by a) diphasic concentration technique, to know the entire parasite spectrum ${ }^{6}$; b) Kato-Katz technique for the identification and quantification of eggs of helminths ${ }^{9}$, and c) by agar-plate coproculture for the detection and identification of STH species with free-living larvae ${ }^{10}$. Considering that eggs of different hookworm species are difficult to distinguish and can be confused with those of hookworm-like species, to avoid this, proper identification can be made after hatching the eggs and cultivating larvae ${ }^{11}$.

Ethics statement: Written informed consent was obtained from each parent or tutor before sampling. The research protocol was reviewed and approved by the local authorities and by the bioethics committee of the University Miguel Hernández de Elche, Alicante, Spain (record num: DF-MPA-001-11).

Molecular characterization: DNA extraction was done from the pellet obtained after centrifugation of larvae culture preserved in ethanol 70\% (6.000 rpm in a bench top microcentrifuge for 10 minutes) after washing with PBS 1\% twice. DNA extractions were done with the commercial kit QIAamp DNA Mini Kit (Quiagen ${ }^{\circledR}$, Barcelona, Spain) following the manufacturer's instructions. Finally, the DNA were eluted in $50 \mu \mathrm{l}$ of elution buffer provided with the kit. PCR were done with oligonucleotide primers NC1 and NC2 designed to regions of the $5.8 \mathrm{~S}$ and $28 \mathrm{~S}$ respectively, to amplifying a 450-580 bp region that found to be conserved across some helminthes groups ${ }^{12}$. Direct sequencing of the PCR products was performed with NC1 and NC2 primers using the Big-Dye Terminator Cycle Sequencing Ready Reaction Kit V3.1 and the automated sequencer "3730 DNA analyzer" (Applied Biosystems, Foster City, CA). Sequences obtained were analyzed and edited using MEGA 6 software (Molecular Evolutionary Genetic Analysis)13 and compared to GenBank ${ }^{\circledR}$ database (U.S. National Library of Medicine, Bethesda, USA).

\section{Results}

In the 497 faecal samples analyzed by Kato-Katz, in 159 samples $(32 \%$; IC95\%: 0.2-0.3) we found the presence of eggs that were similar to those of hookworm (Figure 1A), named as hookworm-like eggs (Figure 1B and 1C). A total of 82 (51.1\%; IC95\%: 43.5-59.5) were from males and 77 (48.4\%; IC95\%: 40.4-56.4) were from females, mostly belonging to children who aged 9 years old (16.4\%; IC95\%: 10.9-23.0).

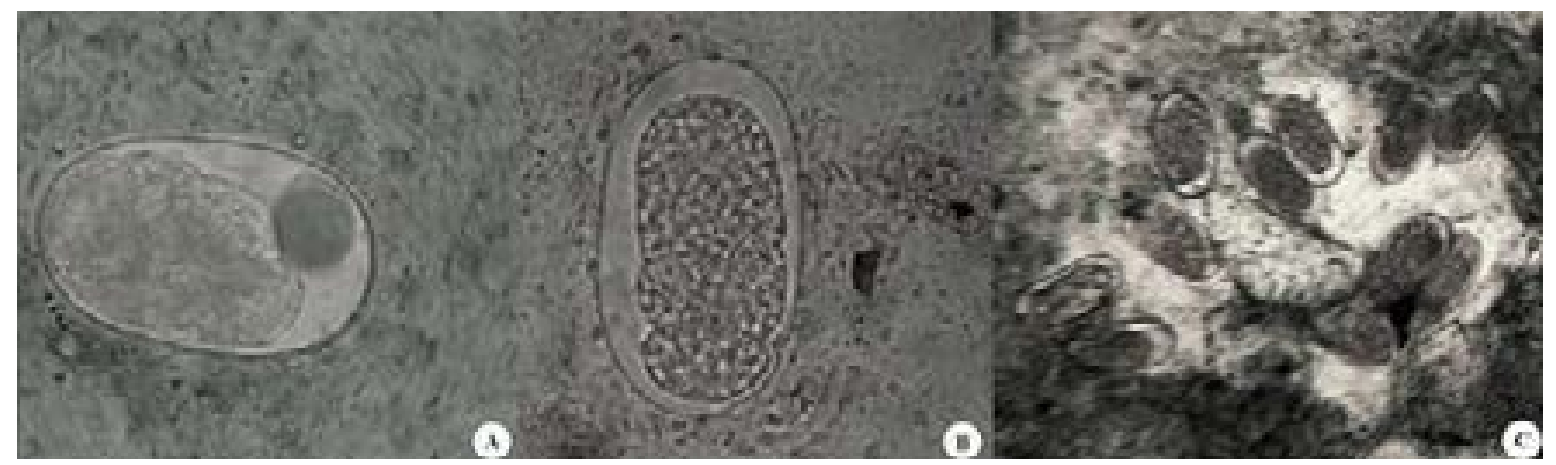

Figure 1. Hookworm egg in Kato-Katz technique (x400) (A); Hookworm-like egg in Kato-Katz slide $(x 400)(B)$, Hookworm-like eggs in Kato-Katz slide $(\mathbf{x 1 0 0 )}(\mathrm{C})$.

Applying the diphasic concentration technique in the 159 samples with hookworm-like eggs, only 2 samples $(1.2 \%$; IC95\%: 0.1-4.4) presented the typical oval round ends of hookworm egg, with a range of size of 62.5 to $67.2 \mu \mathrm{m}$ x 40 to $42.5 \mu \mathrm{m}$ (average size: $65.0 \mathrm{x} 41.6 \mu \mathrm{m}$ ) (Figure 2A). 


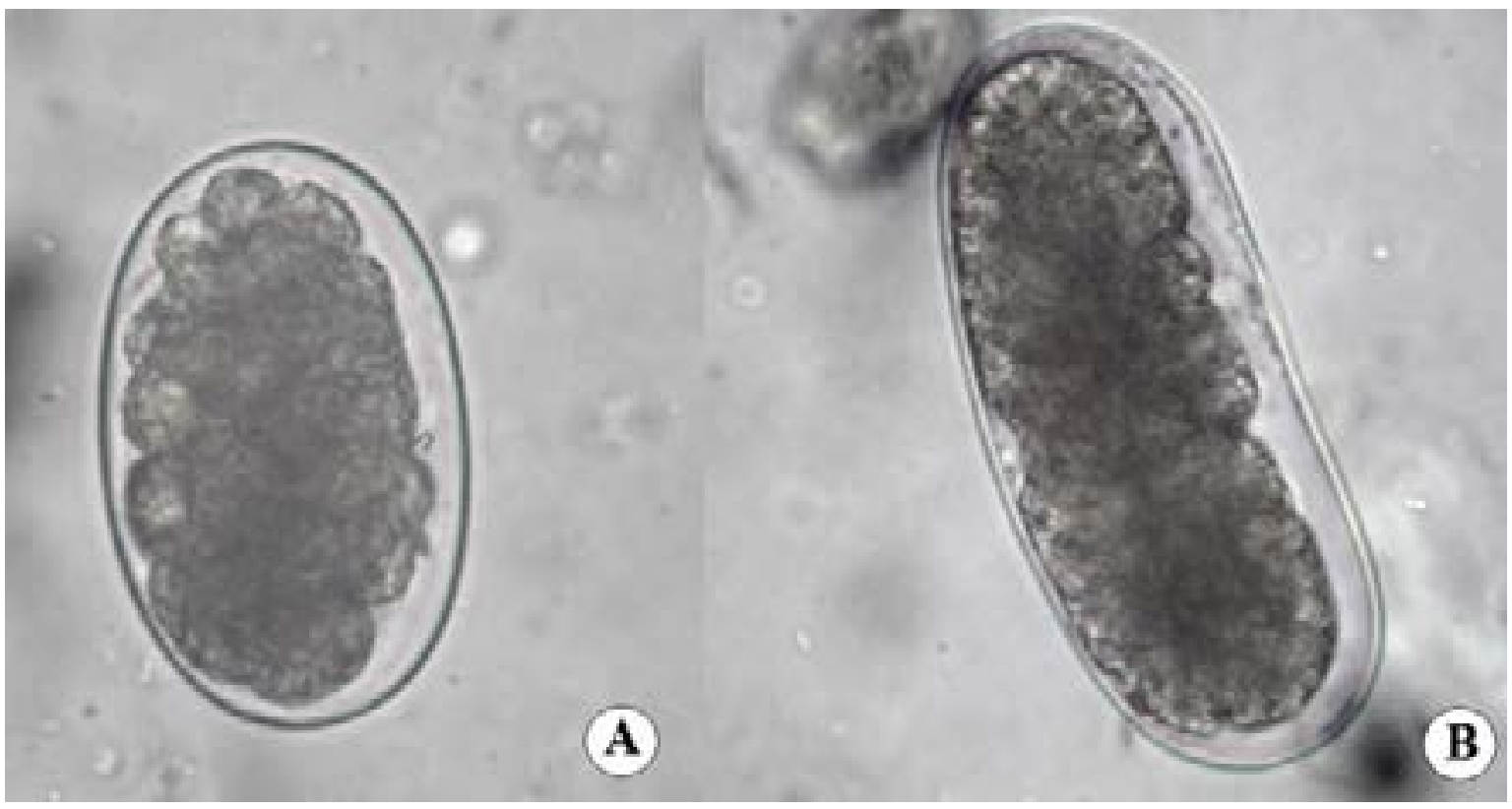

Figure 2. Hookworm egg (Diphasic concentration technique $x$ 400) (A); Hookworm-like egg (Diphasic concentration technique x 400) (B).

In the remaining 157 samples (98.7\%; IC95\%: 95.599.8), hookworm-like eggs with a slightly concave side and with range of size of 85.0 to $92.5 \mu \mathrm{m} \times 35.0$ to 40.0 $\mu \mathrm{m}$ (average size: $89.1 \times 36.8 \mu \mathrm{m}$ ) were observed (Figure 2B). Moreover, female adult worms were also observed in two of those 157 samples (Figure 3).

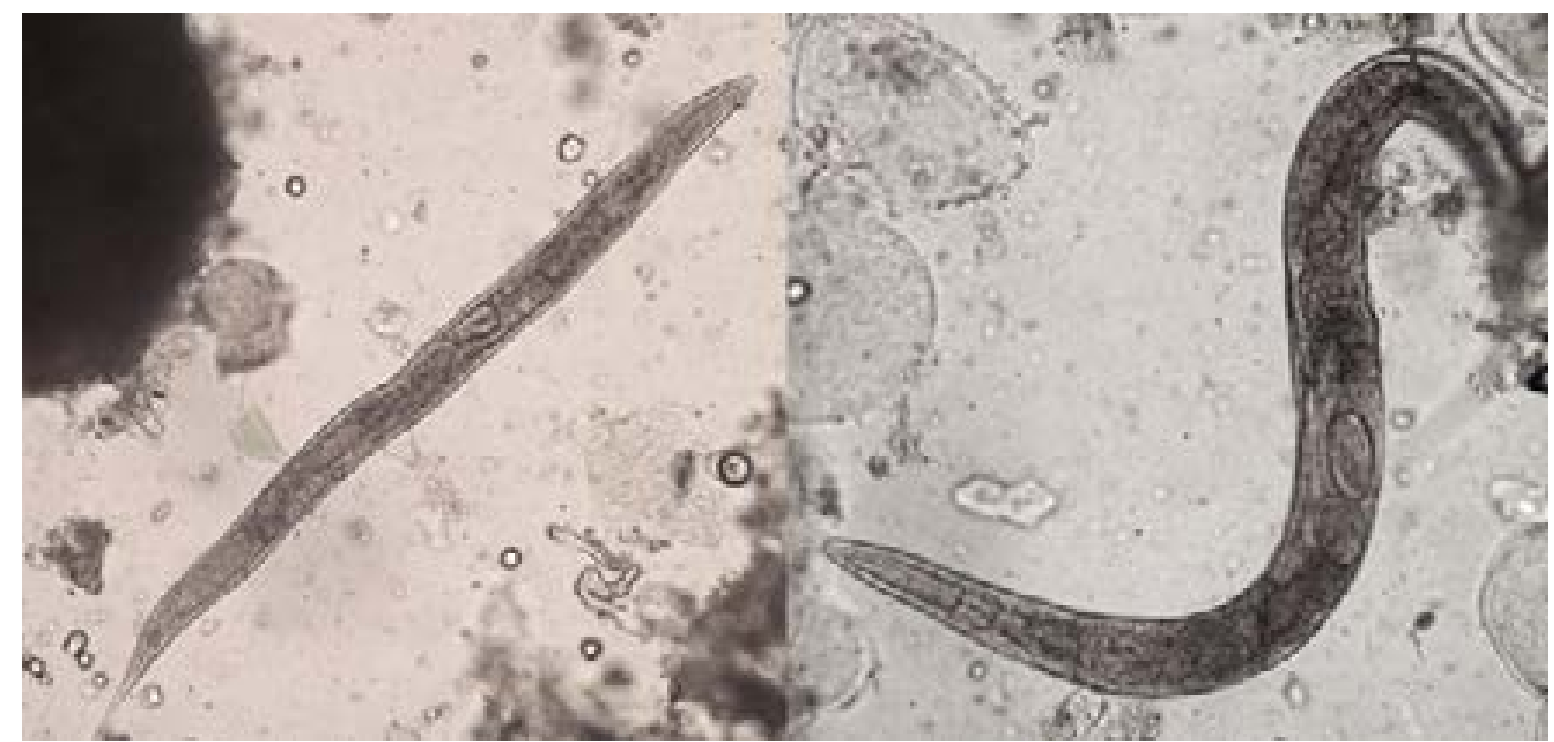

Figure 3. Adult worms of C. elegans (Diphasic concentration technique $x$ 100)

Comparing the results obtained by both techniques, and considering the diphasic concentration technique more precise than the Kato-Katz, the specificity $(68 \%)$, sensibility $(100 \%)$ and positive $(1.3 \%)$ and negative $(100 \%)$ predictive values intended for Kato-Katz, revealed its limitations in the correct identification of hookworm eggs.

Finally, sequence of the 500bp band obtained by PCR, from the larvae of the $159 \mathrm{Kato}-\mathrm{Katz}$ positive-samples agar-plate culture (Figure 4), matched with Caenorhabditis elegans, a free-living nematode. 


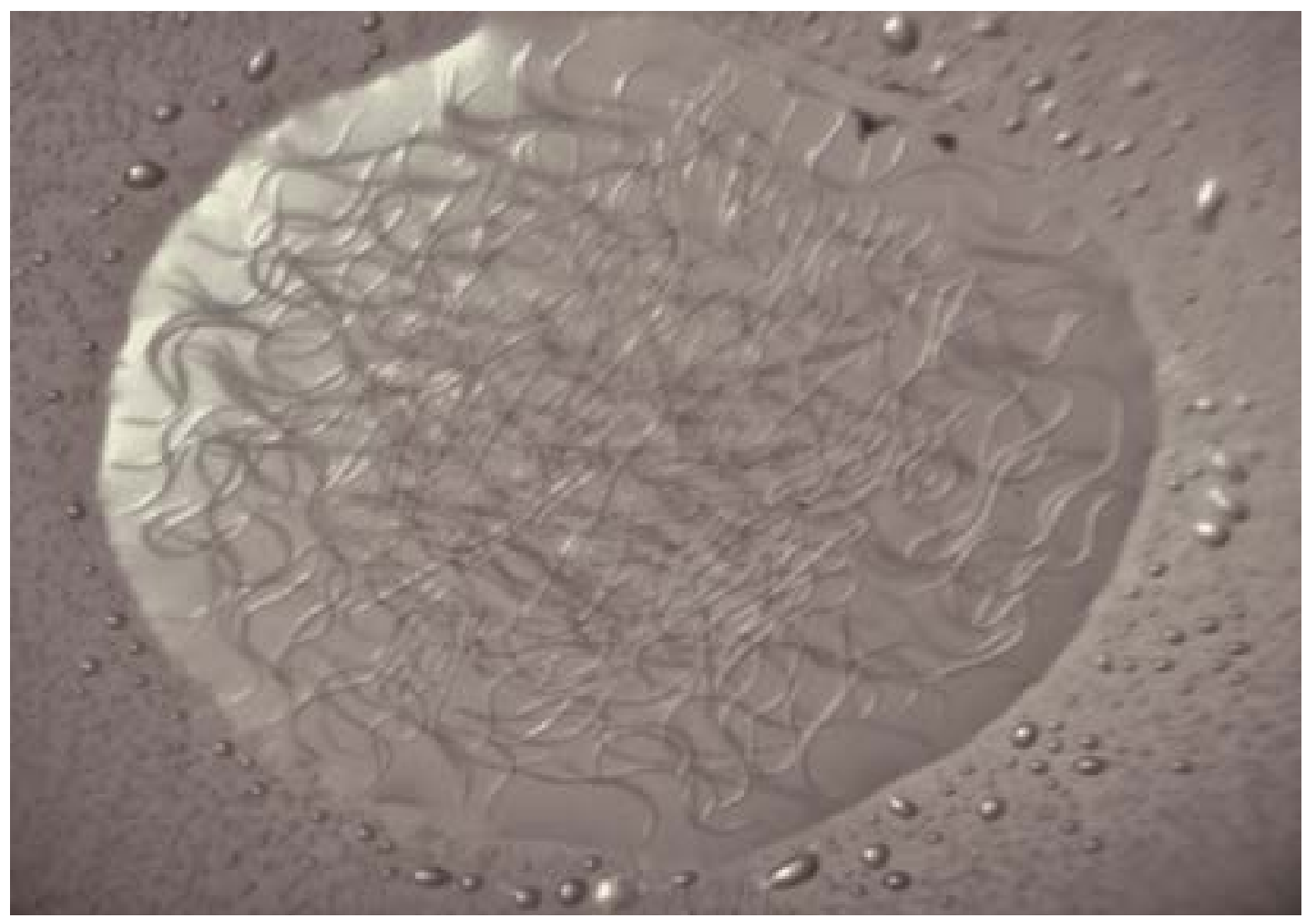

\section{Figure 4. Larvae of $\mathrm{C}$. elegans in water drop from the coprocultive (vision through electronic loupe).}

\section{Discussion}

A brief review of other studies showed frequent problems in the identification of hookworm eggs by microscopy. It is often reported as misdiagnosis of hookworm eggs with other nematode species. Some researchers ${ }^{14-17}$ have found eggs that initially were classified as hookworm eggs, but with further analysis it was proved that these eggs were of Trichostrongylus spp. Other studies $^{15,18}$ also affirm that confusion between hookworms eggs and Oseophagostum bifurcum it is possible because of similarity of eggs, because of that, a study ${ }^{19}$ used a multiplex real-time PCR to achieve the accurate distinction between hookworms and O. bifurcum. In addition, another study ${ }^{15}$ affirms that hookworm eggs can also be confused with Ternidens deminutus eggs. Also happens that eggs similar to those of hookworms could be from plant parasitic nematode eggs, as it is the case of Meloidogyne spp. ${ }^{20}$ and Heterodera spp. ${ }^{15,21-23}$ Finally, confusion may also occur with mite eggs ${ }^{15,23,24}$ and even artefacts such as plant cells ${ }^{22,23}$.

In the present work, it was observed that eggs of the free-living nematode C. elegans, which can be found in humus, compost heaps, garden soil, flowers and fruits who have fallen on the ground ${ }^{25}$, can lead to a misclassification with hookworm eggs mainly by Kato-Katz.
The limitations found in this Kato-Katz technique in the correct identification of hookworm eggs, highlight the use of several diagnostic techniques, as can be the diphasic concentration and/or PCR, in order to perform an adequate diagnosis of hookworm.

Since the stool samples studied in the present work proceeds from northern Rwanda, a rural tropical area, it is important to keep in mind the high incidence of Caenorhabditis species in these wet tropical areas ${ }^{26}$.

\section{Conclusion}

The diagnosis of human hookworm eggs only by the use of Kato-Katz technique, can lead to the occurrence of false positive results, due to similarity with those of other free-living worms that may be present in the stools. Because of this, to give appropriate instructions for a correct stool sample collection, without any contact with soil, land, animal faeces, etc., it is extremely important. Moreover, the use of several diagnostic techniques, to achieve a correct diagnosis of hookworm species, results evident.

Our results, together with the brief bibliographic review, emphasize the importance not only of the correct identification of the structures found in faecal samples, but also the importance of avoiding sample contamina- 
tion from soil material, even when people were instructed correctly about how to proceed with the collection of the samples.

\section{References}

1. Brooker S, Clements ACA, Bundy DAP. Global epidemiology, ecology and control of soil-transmitted helminth infections. Advances in Parasitology 2006; 62 221-261.

2. Staudacher O, Heimer J, Steiner F, et al. Soil-transmitted helminths in southern highland Rwanda: associated factors and effectiveness of school-based preventive chemotherapy. Tropical Medicine and International Health 2014; 19 (7):812-824.

3. Goodman D, Haji HJ, Bickle QD, et al. A Comparison of methods for detecting the eggs of Ascaris, Trichuris, and Hookworm in infant stool, and the epidemiology of infection in Zanzibari infants. The American Journal of Tropical Medicine and Hygiene 2007; 76 (4): 725-731.

4. Knopp S, Mgeni AF, Khamis IS, et al. Diagnosis of soil-transmitted helminths in the era of preventive chemotherapy: effect of multiple stool sampling and use of different diagnostic techniques. PLoS Neglected Tropical Diseases 2008; 2 (11): e331.

5. Tarafder MR, CarabinH, Joseph L, Balolong E, Olveda R, McGarvey ST. Estimating the sensitivity and specificity of Kato-Katz stool examination technique for detection of hookworms, Ascaris lumbricoides and Trichuris trichiura infections in humans in the absence of a 'gold standard'. International Journal for Parasitology 2010; 40 (4): 399-404.

6. World Health Organization (WHO): Bench aids for the diagnosis of intestinal parasites, 1994.

7. Montresor A, Crompton DWT, Hall A, Bundy DAP, Savioli LA. Guidelines for the evaluation of soil-transmitted helminthiasis and schistosomiasis at community level. Geneva: World Health Organization 1998; pp.148.

8. Dacombe RJ, Crampin AC, Floyd S, et al. Time delays between patient and laboratory selectively affect accuracy of helminth diagnosis. Transactions of the Royal Society of Tropical Medicine and Hygiene 2007; 101 (2):140-145.

9. Kato K, Miura M. Comparative examinations. Japanese Journal of Parasitology 1954; 3 (5).

10. Arakaki T, Iwanga M, Kinjo F, Saito A, Asato R, Ikeshiro T. Efficacy of agar-plate culture in detection of Strongyloides stercoralis infection. The Journal of Parasitology 1990; 76: 425-428.
11. Jozefzoon LM, Oostburg BF. Detection of hookworm and hookworm-like larvae in human fecocultures in Suriname. The American Journal of Tropical Medicine and Hygiene 1994; 4: 501-505.

12. Gasser RB, Chilton NB, Hoste H, Beveridge I. Rapid sequencing of rDNA from single worms and eggs of parasitic helminths. Nucleic Acids Research1993; 21 (10): 2525-2526.

13.Tamura K, Stecher G, Peterson D, Filipski A, Kumar S. MEGA6: molecular evolutionary genetics analysis version 6.0. Molecular biology and Evolution 2013; 30 (12):2725-2729.

14. Ralph A, O'Sullivan MVN, Sangster NC, Walker JC. Abdominal pain and eosinophilia in suburban goat keepers-trichostrongylosis. Medical Journal of Australia 2006; 184 (9): 467-469.

15. Thibert JB, Guiguen C, Gangneux JP. Diagnostic microscopique différentiel des oeufs de type Ankylostomidé: à propos d'un cas de'Trichostrongyloïdose. $A n$ nales de Biologie Clinique 2006; 64 (3): 281-285.

16. Yong TS, Lee JH, Sim S, et al. Differential diagnosis of Trichostrongylus and Hookworm eggs via PCR using ITS-1 sequence. The Korean Journal of Parasitology 2007; 45 (1): 69-74.

17. Bradbury RS, Males C, Goldmisd JM. An unusual helminth infection in a South East Asian refugee. $A n$ nals of the Australasian College of Tropical Medicine 2010; 11 (1): 16-18.

18. Goldsmid JM. The differentiation of Ternidens deminutus and hookworm ova in human infections. Transactions of the Royal Society of Tropical Medicine and Hygiene 1968; 62 (1):109-116.

19. Verweij JJ, Brienen EAT, Ziem J, Yelifari L, Polderman AM, Van Lieshout L. Simultaneous detection and quantification of Ancylostoma Duodenale,Necator Americanus, and Oesophagostomum bifurcum in fecal samples using multiplex Real-Time PCR. The American Journal of Tropical Medicine and Hygiene 2007; 77 (4): 685690.

20. Cantos GA, Dutra RL, Benedet NS. Estudo de ovos de Meloidogyne Spp em fezes humanas. News Lab 2004; 65: 132-36.

21. Sandground JH. Oxyuris incognita Or Heterodera radicicola? The Journal of Parasitology 1923; 10 (2): 92-94. 22. Colmer-Hamood JA. Fecal microscopy artifacts mimicking ova and parasites. Lab Medicine 2001; 32 (2): 80-84.

23. Ash LR, Orihel TC. Atlas de Parasitología Humana. 5th ed. Editorial Médica Panamericana, Argentina 2010. 
24. Werneck JS, Carniato T, Gabriel A,Tufik S, Andrade SS. Mites in clinical stool specimens: potential misidentification as helminth eggs. Transactions of the Royal Society of Tropical Medicine and Hygiene 2007; 101 (11): 11541156.

25. Barrière A, Félix MA. Isolation of C. elegans and related nematodes. WormBook: The Online Review of C. elegans Biology (2014). Available at:http://www. ncbi.nlm.nih.gov/books/NBK19764/. Accessed August 2014.

26. Félix MA, Jovelin R, Ferrari C, et al. Species richness, distribution and genetic diversity of Caenorhabditis nematodes in a remote tropical rainforest. $B M C$ Evolutionary Biology 2013; 13: 10. 